\title{
Multifunctional Nanoparticles for Medical Applications
}

\author{
Sofia Dembski ${ }^{1,2}$, Christine Schneider ${ }^{2}$, Marion Straßer ${ }^{1}$, Bastian Christ ${ }^{2}$, Joern Probst ${ }^{1}$ \\ ${ }^{1}$ Fraunhofer Institute for Silicate Research ISC \\ Neunerplatz 2, 97082 Wuerzburg, Germany \\ sofia.dembski@isc.fraunhofer.de; marion.strasser@isc.fraunhofer.de \\ ${ }^{2}$ Translational Center Wuerzburg "Regenerative therapies in oncology and musculoskeletal diseases" \\ Roentgenring 11, 97070, Wuerzburg, Germany \\ bastian.christ@igb.fraunhofer.de; joern.probst@isc.fraunhofer.de
}

\section{Extended Abstract}

Well-tailored multifunctional nanoparticles (NPs), which are in the focus of our R\&D work, play a major role in the development of future-oriented, advanced functional materials for life science applications such as in contrast agents for medical imaging, for in vitro and in vivo diagnostics, in drug delivery as well as in tissue engineering. Inorganic-organic biohybrid NPs in particular are considered to be important for the development of smart materials and novel technologies for medical applications.

We prepare our NPs by wet-chemical methods: sol-gel, precipitation or hydrothermal synthesis. Our preferred particle materials are inorganic and hybrid materials, e.g. materials based on silicate, calcium fluoride and phosphate, TiO2 and iron oxide[1,2]. To ensure multifunctionality, different approaches are taken: the labelling of the NP matrix with organic dyes or lanthanide ions, as well as the combination of various materials by core-shell NP design. Typical core materials are iron oxide or amorphous silica, typical shell materials are pure silica, organic dye doped silica, Zn2SiO4:Mn2+, Ca10(PO4)6OH:Eu3+, or CaF2:Eu3+[3,4]. The structure, size, and composition of these NPs can easily be controlled to tailor their chemical and physical properties. The influence of the composition and structure of the NP is studied by optical absorption and photoluminescence spectroscopy. Characterization is done by conventional methods such as dynamic light scattering (DLS), transmission electron microscopy (TEM), or X-ray diffraction analysis (XRD). The resulting NPs are subsequently modified with various chemical functionalities such as amine and carboxyl functions using conventional functionalization methods such as silanization. Furthermore, surface modified NPs are covalently attached to biological moieties, e.g. antibodies, nucleobases, or peptides, by means of standard bioconjugation methods [5].

The great potential of novel NPs as assay labels for immunodetection, contrast agents for medical imaging or tools for tumor therapy has been demonstrated in different research projects [6].

In close collaboration with the Fraunhofer translational center "Regenerative therapies in oncology and musculoskeletal diseases", a special application of NPs for in vitro and in vivo monitoring of wound healing process is investigated. To this end, artificial wound models, which help to increase the effectiveness of a medication by testing it in an earlier stage to significantly reduce the number of medications selected for clinical tests, have been developed. In general, the 3D tissue models can be used as a preliminary stage to animal experiments for investigations of functional parameters like the distribution and metabolization of substances in different tissue layers.

The demographic change in the industrialized countries manifests itself in a constantly growing number of humans in need of care and, at the same time, a declining number of available care forces. This effect is strengthened by the fact that cost-intensive illnesses such as cardiovascular diseases, diabetes, dementia, cancer diseases and chronic wounds are growing in numbers with increasing age of the population. This predictable dramatic development can only be countered with improved pre-diagnostics, a marked cost reduction in therapeutic treatment through better efficiency and extended self-care by patients. Here, we present the development and evaluation of economical and innovative therapy procedures for chronic skin diseases, which enable patients to check whether their chronically open wounds are healing. Central elements in the development phase are modifiable in-vitro skin models, biodegradable CE-certified wound dressings, implementation of immunotherapeutics in the wound dressing, targeted nanoparticles for smart diagnostics of biomarkers 
in the wound exudate and imaging of the healing process, and sterilizable fluid actuators integrated in the wound dressing for oxygenation, sampling, and vacuum-assisted closure (V.A.C.) therapy.

\section{References}

[1] K. Mandel and M. Straßer "Surfactant free superparamagnetic iron oxide nanoparticles for stable ferrofluids in physiological solutions," Chem. Com., vol. 51, pp. 2863-2866, 2015.

[2] K. Mandel and C. Kolb "Size controlled iron oxide nano octahedra obtained via sonochemistry and natural ageing," Colloids Surf. A: Physicochemical and Engineering Aspects, vol. 457, pp. 27-32, 2014.

[3] S. Dembski and S. Rupp "Luminescent silicate core-shell nanoparticles: Synthesis, functionalization, optical, and structural properties," J. Colloid Interface Sci., vol. 358, pp. 32-38, 2011.

[4] S. Dembski and S. Rupp "Synthesis and optical properties of luminescent core-shell structured silicate and phosphate nanoparticles," Opt. Mater., vol. 33, pp. 1106-1110, 2011.

[5] F. Kampmeier and M. Ribbert "Site-Specific,Covalent Labeling of Recombinant Antibody Fragments via Fusion to an Engineered Version of 6-O-Alkylguanine DNA Alkyltransferase," Bioconjugate Chem., vol. 20, pp. 1010-1015, 2009.

[6] Fraunhofer ISC, Bioactive Materials, [Online]. Available: http://www.isc.fraunhofer.de/focal-areas/theranostic/aboutus/?L=1 